\title{
Controlling the Ratchet Effect for Cold Atoms
}

\author{
Anatole $\operatorname{Kenfack}^{(a)}$, Jiangbin Gong ${ }^{(b)}$, and Arjendu K. Pattanayak ${ }^{(c)}$ \\ (a) Max-Planck-Institut für Physik Komplexer Systeme, \\ Nöthnitzer Strasse 38, D-01187 Dresden, Germany \\ (b) Department of Physics and Center for Computational Science and Engineering, \\ National University of Singapore, 117542, Republic of Singapore \\ (c) Department of Physics and Astronomy, Carleton College, Northfield, Minnesota 55057, USA
}

(Dated: November 3, 2018)

\begin{abstract}
Low-order quantum resonances manifested by directed currents have been realized with cold atoms. Here we show that by increasing the strength of an experimentally achievable delta-kicking ratchet potential, quantum resonances of a very high order may naturally emerge and can induce larger ratchet currents than low-order resonances, with the underlying classical limit being fully chaotic. The results offer a means of controlling quantum transport of cold atoms.

PACS numbers: 05.45.-a, 32.80.Qk, 05.60Gg
\end{abstract}

The ratchet effect, i.e., the possibility to derive directed transport without bias in periodic systems with broken symmetries, was originally proposed by Feynman. This effect, prohibited in systems at equilibrium by the second law of thermodynamics [1], has recently gained renewed interest [2, 3] as a model for the physics of molecular motors [4]. Directed transport is possible when particles are driven out of equilibrium and relevant spatio-temporal symmetries are broken [5]. This has motivated the construction of nanoscale devices in which artificial ratchets may serve as new electrons pumps, molecular switches and particle selectors, among other applications [4, 6, 7]. Other studies have shown that when the noise is absent, its role can be replaced by deterministic chaos induced by the inertial term 8]. In such inertial ratchets, the issue of current reversal was intuitively addressed [9] and later carefully reformulated [10]. Purely Hamiltonian ratchets, where noise and friction are eliminated, have received notable attention as well [5, 11].

Besides these classical ratchets, quantum Hamiltonian ratchet effects arising from purely unitary evolution is also possible. These are very important, for example, for the design of coherent nanoscale devices [12]. Exploring quantum coherence phenomena in chaotic Hamiltonian ratchets hence becomes necessary. The quantum deltakicked rotor (QKR), a paradigm of quantum chaos [13], is a convenient and experimentally realizable model for such explorations, possessing dynamical localization [14], quantum accelerator modes [15], tunneling [12, 16], as well as quantum resonances 17, 18, 19, 20, 21, 22, 23. The "quantum ratchet accelerator", where the coherent ratchet current accelerates linearly, was first studied with a modified QKR and later in the kicked Harper model [24].

Since the pioneering experiment of cold-atom ratchets [25], new designs looking for ratchet effects in nonlinear Hamiltonian systems [26] have emerged. Motivated by the first experimental realizations of sawtooth-like asymmetric potentials [27, 28] as well as quantum reso- nance ratchets 29], in this Letter we revisit the quantum flashing ratchet model in Ref. [30], with the perspective of detecting and controlling quantum resonance dynamics of very high orders. The ultimate goal is to help design powerful means for the coherent control of the dynamics of cold atoms with driven but dissipationless optical lattices. For other cold-atom control scenarios using also Hamiltonian ratchet effects, see Refs. [31, 32].

A quantum resonance occurs when the flashing period is commensurate with the recoil frequency and is related to the arithmetic nature of the effective Planck constant $\widetilde{\hbar}$ of kicked systems, occuring specifically if

$$
\widetilde{\hbar}=4 \pi r / s,
$$

with $r, s$ being mutually prime integers. Cases with small $s$ and large $s$ values can be called low-order quantum resonance (LOQR) and high-order quantum resonance (HOQR), respectively. We show below that HOQRs can manifest themselves strongly in the ratchet current behavior, with their corresponding classical phase space being fully chaotic. This further enhances the view that quantum control techniques can be applied to classically chaotic systems 33].

The system we consider is described, in dimensionless units, by the following Schrödinger equation 30]

$$
i \widetilde{\hbar} \frac{\partial \psi}{\partial t}=-\frac{\widetilde{\hbar}^{2}}{2} \frac{\partial^{2} \psi}{\partial x^{2}}+v(x) \sum_{l=0}^{\infty} \delta(t-l) \psi
$$

where $x$ is the position, and the potential $v(x)=$ $K[\sin (x)+\alpha \sin (2 x)]$ is assumed to be periodically flashed off and on with delta kicks. Here $t$ is the time variable and $l$ an integer that counts the number of kicks. By superimposing a conventional standing wave potential of $\lambda / 2$ spatial periodicity with a fourth-order lattice potential of $\lambda / 4$ periodicity, such a dissipationless ratchet potential $v(x)$ has been successfully engineered [27, 28]. The scheme, satisfying the Raman-Nath transition processes 27, 28, 34], uses three level atoms with two stable 
ground states and one electronically excited state (for more experimental details, see Ref. [27]). Our results below can thus be experimentally verified by taking parameters from Refs. 27, 28] for alkali-metal atoms such as Rb and Cs. Note that $\alpha=V_{2} / V_{1}$ and $K=V_{1} / 2$, where $V_{1}$ and $V_{2}$ denote the potential depths of the lattice harmonics $\lambda / 2$ and $\lambda / 4$, respectively. The parameter $\alpha$ controls the skewness of the potential. With $\alpha \in(0,0.5]$ the sawteeth of the potential lean left, stimulating the transport to the right for classical diffusive motion. The familiar rotor potential can be recovered for $\alpha=0$. For this temporally-symmetric system, the ratchet effect is only possible for broken spatial symmetry, i.e., for $\alpha \neq 0$. We use scaled units here with both the spatial $L$ and the temporal $T$ periods set to unity. The quantum nature of the system is in the effective Planck constant $\widetilde{\hbar}=8 \omega_{R} T$, which varies as one adjusts the pulsating period $T$. Here $\omega_{R}=\hbar k_{L}^{2} / 2 m$ is the recoil frequency of the applied laser field, with $m$ the atom mass and $k_{L}$ the photon wave number that makes up a lattice period of $\left(2 k_{L}\right)^{-1}$ for the optical potential.

The quantum map of the above delta-kicked ratchet model is given by $\hat{U}=\exp \left(-i \widetilde{\hbar} \hat{k}^{2} / 2\right) \exp (-i P v(\hat{x}))$, where $\hat{x}$ and $\hat{k}=-i \frac{\partial}{\partial x}$ represent the position and the wave number operators, respectively. Here we have also defined $P=K / \widetilde{\hbar}$ for later use. All computational examples presented below are for fixed skewness parameter $\alpha=0.3$.

Quantum resonances have been long studied in kicked rotor systems [17, 18, 19, 20, 21]. A direct observation of quantum resonance dynamics is known to require initial states with long coherence width. This issue is heightened for HOQRs. Very recently non-condensed atoms were used to indirectly observe a particular family of HOQRs 35. However, using Bose-Einstein condensates loaded in optical lattices [23, 29], one can now easily realize initial quantum states whose coherence spans many optical lattice sites, resulting in the observations of the main quantum resonance [29] as well as quantum resonances of relatively low orders 23]. Given this experimental progress we assume below, unless stated otherwise, that the initial wavefunction is homogeneous with zero initial momentum. We then let $\widetilde{\hbar}$ take specific values and explore new quantum resonance effects in the ratchet transport.

The ratchet current, denoted $\langle k\rangle$ below, is defined as the expectation value of $\hat{k}$. Its time dependence is shown in Fig. (1) for several values of the potential strength $P$ and for specific values of $\widetilde{\hbar}$. One clearly sees in Fig. 1(a) that there is no directed transport for $\widetilde{\hbar}=1.001 \pi$ with any value of the potential strength. By sharp contrast, the current acceleration may be strongly favored in one or the other direction as illustrated in Fig. 1(b), (c), and (d), where $\widetilde{\hbar}=0.7 \pi$ for $(r, s)=(7,40), \widetilde{\hbar}=2.625 \pi$ for $(r, s)=$ $(21,32)$, and $\widetilde{\hbar}=1.5 \pi$ for $(r, s)=(3,8)$, respectively.
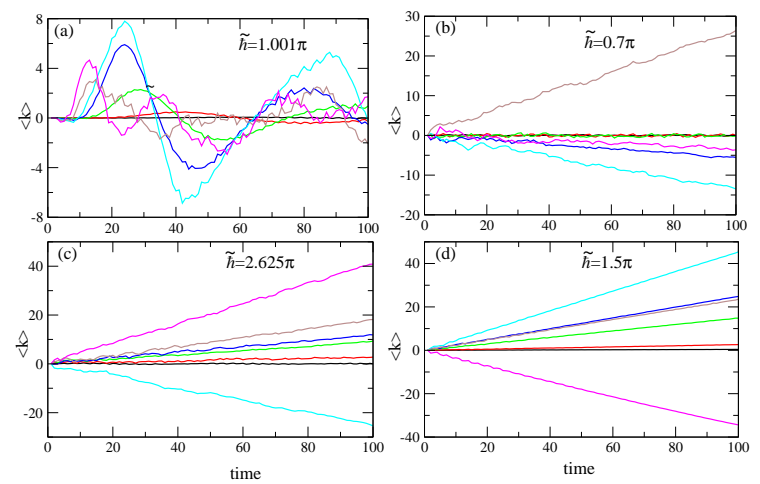

FIG. 1: Time dependence of the ratchet current for different values of $P$ and $\widetilde{\hbar}$. In panels (a), (b) and (d), $P=0.5$ (black), 1.0 (red), 2.0 (green), 3.0 (blue), 4.0 (cyan), 5.0 (brown) and 6.0 (magenta). (a) $\widetilde{\hbar}=1.001 \pi$ and no directed transport occurs for any value of $P$. (b) $\widetilde{\hbar}=0.7 \pi$ with the tuple $(r, s)=$ $(7,40)$; transport occur in either direction, at $P$-dependent acceleration rates. (d) As in (b) except $\widetilde{\hbar}=1.5 \pi ;(r, s)=$ (3,8). In panel (c) $P=1.0$ (black), 2.0 (red), 3.0 (green), 4.0 (blue), 5.0 (cyan), 7.0 (brown) and 8.0 (magenta), with $\widetilde{\hbar}=2.625 \pi ;(r, s)=(21,32)$. (Color online)

Note that cases in Figs. 2(b) and 2(c) represent quantum resonances of very high orders (much higher than those observed in Ref. 23] ). Unlike the ratchet current of a low order resonance (where current reversal may be well understood by noticing that the wavefunction amplitude at a point $x$ at $t=l$ is a coherent sum of the wavefunction amplitudes at other locations at $t=l-1[30]$ ), here a simple explanation of the HOQR current dependence on $P$ is not available. Indeed, an analytical treatment of the ratchet transport associated with those very high order resonances seems very difficult, even for a perturbation theory using very small $\alpha$ [36].
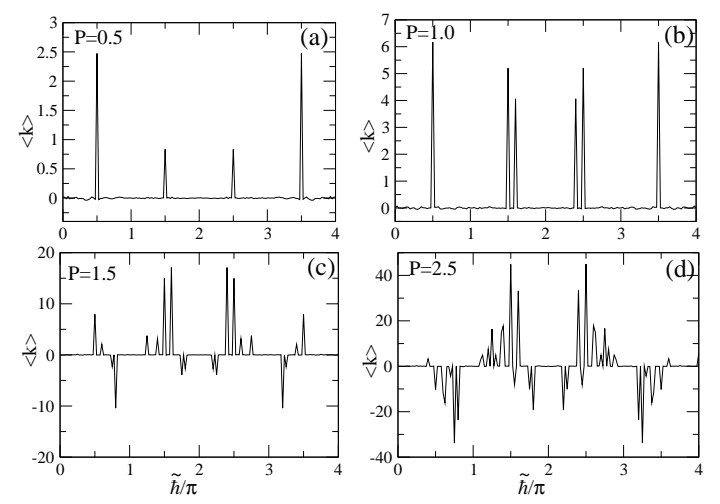

FIG. 2: Ratchet current $\langle k\rangle$ as a function of $\widetilde{\hbar} / \pi$ after 200 kicks, with the potential parameter $\alpha=0.3$, and the potential strength $P$ indicated in each panel. Main resonances appear in (a) with low $P$. As $P$ increases in (b), (c), and (d), full chaos is being developed (see Fig. 3) and significant ratchet currents due to higher-order quantum resonances emerge. 
Figure 2 displays $\langle k\rangle$ after 200 temporal periods as a function of $\widetilde{\hbar}$. In Fig. 2(a) $P=0.5$ and the results correspond to the findings of Ref. [30], showing a net drift at those quantum resonances where $\widetilde{\hbar} / \pi$ is given by halfintegers. As we increase $P$ the system exhibits dramatic changes. For example, when $P=1.0$ in Fig. 2(b) two twin peaks emerge. One also sees, as shown in Fig. 2 (c) and (d), that larger values of $P$ lead not only to a proliferation of peaks, but also to current reversals. As shown in Table I, many of these peaks are found to be associated with very high-order quantum resonances covering a wide range of $(r, s)$. Remarkably, these high-order resonances may yield larger ratchet current acceleration than the main resonances, and in either case the current direction depends on $P$. Note, however, that HOQRs do not always transport better than LOQR (compare, for example, the HOQR at $\hbar / \pi=0.6$ to the LOQR at $\hbar / \pi=0.5,1.5,3.5)$. Figure 2 also shows that relatively small changes in $\widetilde{\hbar}$ can dramatically change the ratchet current, thus experimentally offering a means of isolating different HOQRs. This also suggests that particles with slightly different masses, hence slightly different $\widetilde{\hbar}$ and different $P$ due to an isotope effect, may display qualitatively different kinds of transport. Neglecting at the moment the non-ideal situation in experiments, note that the resonance peaks shown in Fig. (2) are better resolved with increasing kicks since the absolute amplitude of the current peaks is proportional to the number of kicks.

\begin{tabular}{|c|c|c|c|c|c|c|}
\hline$\hbar / \pi$ & 0.6 & 0.7 & 0.75 & 1.125 & 1.55 & 3.3 \\
\hline$(r, s)$ & $(3,20)$ & $(7,40)$ & $(1,16)$ & $(9,32)$ & $(31,80)$ & $(33,40)$ \\
\hline
\end{tabular}

We now comment on the possibility of experimentally observing these HOQR peaks. Using constraints associated with state-of-the-art experiments, we have carried out extensive computations [37], verifying that the aboveobserved HOQR ratchet current can be clearly observed in experiments, at least for time scales of $20-30$ kicks. In particular, the ratchet currents are sufficiently stable when (i) considerable dephasing is present, (ii) a finite pulse-width instead of delta-kicks is considered, and (iii) a realistic quasi-momentum spread in the initial state is considered. For example, for the case of $\widetilde{\hbar}=2.625 \pi$, $P=5.0$ shown in Fig. $1(\mathrm{c})$, we find that the HOQR ratchet current deviates considerably from the ideal case only after about 20 kicks, for a realistic quasi-momentum spread as estimated in Ref. 23]. We have also checked that if a superposition state of momentum (such as in Ref. [29]) is used as the initial state, then a HOQR ratchet current can be also effectively demonstrated without using a bichromatic optical lattice.

Let us now briefly describe the classical dynamics of the model system. With the classical stochasticity pa- rameter or kick strength $K=\widetilde{\hbar} P$, the associated classical map is given by $p_{l+1}=p_{l}-K\left[\cos \left(x_{l}\right)+2 \alpha \cos \left(2 x_{l}\right)\right]$; $x_{l+1}=x_{l}+p_{l+1}$, where $p_{l}$ is the momentum variable conjugated to the coordinate $x_{l}$. Figure 3 displays the classical phase space for $\alpha=0.3$ and for varying $K$. As $K$ increases, the islands initially dominating the phasespace shrink and decrease in number, [see Fig. 3 (a), (b), (c)], until a threshold value $K_{\mathrm{thr}}$, when essentially full chaos is reached. In Fig. 迆(d), $K=0.8 \pi>K_{\mathrm{thr}} \approx 0.75 \pi$, the entire phase space is seen to be chaotic.
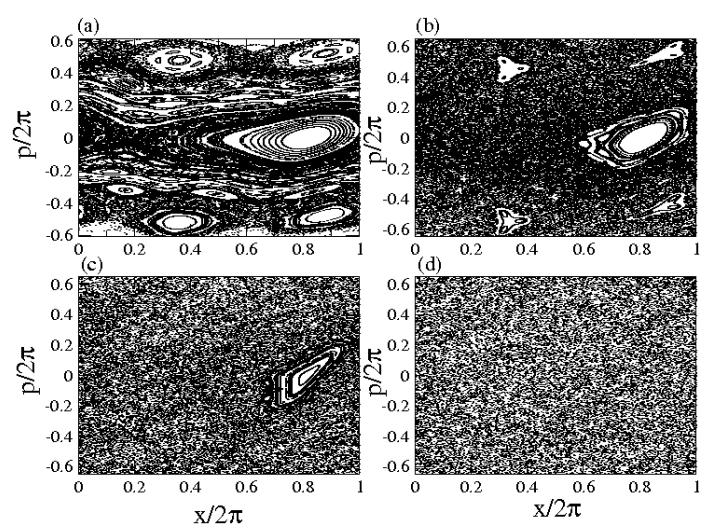

FIG. 3: Classical phase space structures for the kicked ratchet map for $\alpha=0.3$ showing regular islands embedded in the chaotic sea for (a) $K=0.25 \pi$, (b) $K=0.55 \pi$ and (c) $K=$ $0.70 \pi$. In panel (d) $K=0.8 \pi$ and full chaos is reached.

Remarkably, we observe that clear HOQR peaks of the ratchet current emerge only when the classical counterpart is fully chaotic. This observation has been checked by varying $\alpha$ in the range 0 to 10 , with different $K_{\mathrm{thr}}$. Such a connection between a purely quantum phenomenon and a purely classical phenomenon is worthy of some remarks, though a profound explanation may not exist. First, quantum resonances lead to continuous energy bands [17]. A potential of height $P=K / \hbar$ supports only a certain number of energy bands (denoted $n$ ) that are below the potential barrier. As the well deepens, $n$ increases with $K$, specifically $n \propto \sqrt{K}$ as shown in the inset in Fig. 4. For a fixed $\widetilde{\hbar}=K / P$, larger $K$ corresponds to more classical chaos, and leads to more bands that can contribute to transport, whence a HOQR is more likely to be detected. Alternatively, as we increase $K$ for fixed $P, n$ increases, leading to more resonant values $\widetilde{\hbar}$ being available. Both ways, the result is more peaks in the plot of the ratchet current vs $\widetilde{\hbar}$. As such, chaos and HOQRs, both requiring sufficiently large $K$, go hand-in-hand, an interesting result also noticed [35] using other signatures. Since classical chaos arises through the growth of nonlinear resonances [38], its connection with HOQRs might exist at a deeper level, though further work along this line is beyond the scope of this paper. Finally, note that for our Hamiltonian ratchet model, directed transport may occur for $n<2$, albeit being weaker than in cases with 
larger $n$. This is in contrast to dissipative systems [39], where the ratchet effect exists only if there are at least two bands below the barrier.

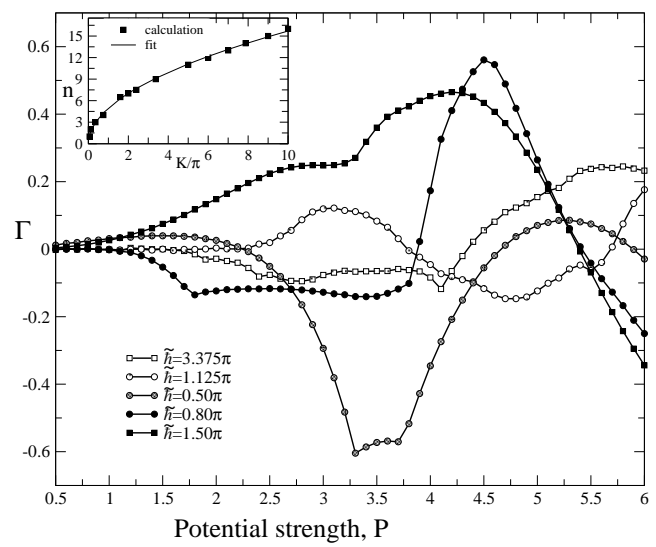

FIG. 4: Acceleration rate $\Gamma$ as a complicated function of the potential strength $P$ for different $\widetilde{\hbar}$. The inset shows the number of Floquet bands below the potential barrier as a function of $K$, fitted empirically as $n \propto \sqrt{K}$.

Finally, to motivate further theoretical work, we show in Fig 4 the average current acceleration rate $\Gamma \equiv\langle k\rangle / l$ within $l=100$ kicks, as a function of $P$, for $\widetilde{\hbar}$ corresponding to a few HOQRs as well as LOQRs. The size and direction of $\Gamma$ are both seen to be tunable with $P$, with their $P$-dependence varying markedly with $\widetilde{\hbar}$.

In summary, we have shown the important role of quantum resonances of very high orders in a QKR-based quantum ratchet. The associated transport can be manipulated by use of these high-order quantum resonances. We also observe, and partially explain, that the transport associated with HOQRs become important only if the associated classical phase space is fully chaotic. The results are of great experimental interest because (i) they offer a ratchet acceleration mechanism previously not noticed and (ii) suggest a new means of detecting the intriguing quantum high-order resonances in QKR systems. This study should help design new means of controlling the dynamics of cold atoms in pulsed optical lattices.

Fruitful discussions with Kamal P. Singh and J.M. Rost are acknowledged. A. K. acknowledges supports from the Alexander von Humboldt foundation as well as the Max-Planck Gesellschaft through the Reimar Lüst fund (2005). J. G. was supported by the start-up fund (WBS grant No. R-144-050-193-101 and No. R-144-050193-133), and the NUS "YIA" fund (WBS grant No.: R-144-000-195-123), both from the National University of Singapore.

[1] R. P. Feynman et al., The Feynman Lectures on Physics (Addison-Wesley, Reading, MA, 1966), Vol.1, Chap.46.
[2] R. D. Astumian and P. Hänggi, Physics Today 55 (11), 33 (2002).

[3] P. Reimann, Phys. Rep. 361, 57 (2002).

[4] F. Jülicher et al., Rev. Mod. Phys. 69, 1269 (1997).

[5] S. Flach et al., Phys. Rev. Lett. 84, 2358 (1997); O. Yevtushenko et al., Europhys. Lett. A54, 141 (2001); R. Gommers et al, Phys. Rev. Lett. 96, 140604 (2006).

[6] R. D. Astumian, Science 276, 917 (1997).

[7] J. Lehmann et al., Phys. Rev. Lett. 88, 228305 (2002).

[8] P. Jung et al., Phys. Rev. Lett. 76, 3436 (1996).

[9] J. L. Mateos, Phys. Rev. Lett. 84, 258 (2000).

[10] A. Kenfack, S. M. Sweetnam, and A. K. Pattanayak, Phys. Rev. E 75056215 (2007).

[11] T. Dittrich et al., Ann. Phys. (Leipzig) 9, 755 (2000); H. Schanz et al., Phys. Rev. Lett. 87, 070601 (2001); S. Denisov et al., Phys. Rev. E 66, 046203 (2002); T. Cheon et al., J. Phys. Soc. Jpn. 72, 1087 (2003).

[12] P. Reimann, M. Grifoni, and P. Hänggi, Phys. Rev. Lett. 79, 10 (1997); P. Reimann, and P. Hänggi, Chaos 8, 629 (1998).

[13] Quantum Chaos: Between Order and Disorder, ed. by G. Casati and B. Chirikov (Cambridge Univ. Press, Cambridge, England, 1995).

[14] S. Fishman et al., Phys. Rev. Lett. 49, 509 (1982).

[15] S. Schlunk et al., Phys. Rev. Lett. 90, 124102 (2003).

[16] H. Linke et al., Science 286, 2314 (1999).

[17] F. M. Izrailev and D. L. Shepelyanskii, Theor. Math. Phys.43, 553 (1980).

[18] A. J. Daley and A. S. Parkins, Phys. Rev. E 66, 056210 (2002).

[19] F.L. Moore et al., Phys. Rev. Lett. 75, 4598 (1995).

[20] G. Duffy et al., Phys. Rev. E 70, 056206 (2004).

[21] S. Wimberger et al., Phys. Rev. Lett. 92, 084102 (2004).

[22] I. Dana and D. L. Doforeev, Phys. Rev. E 73, 026206 (2006).

[23] C. Ryu et al., Phys. Rev. Lett. 96, 160403 (2006).

[24] J. Gong and P. Brumer, Phys. Rev. E 70, 016202 (2004); Phys. Rev. Lett. 97, 240602 (2006).

[25] C. Mennerat-Robilliard et al., Phys. Rev. Lett. 82, 851 (1999).

[26] T. S. Monteiro et al., Phys. Rev. Lett. 89, 194102 (2002).

[27] G. Ritt et al., Phys. Rev. A 74, 063622 (2006)

[28] T. Salger et al., Phys. Rev. Lett. 99, 190405 (2007).

[29] M. Sadgrove et al., Phys. Rev. Lett. 99, 043002 (2007); I. Dana et al, arxiv: physics/0706.0871.

[30] E. Lundh and M. Wallin, Phys. Rev. Lett. 94, 110603 (2005).

[31] S. Denisov et al., Phys. Rev. A 75, 063424 (2007).

[32] J. Gong, D. Poletti, and P. Hanggi, Phys. Rev. A 75, 033602 (2007).

[33] J. Gong and P. Brumer, Ann. Rev. Phys. Chem. 56, 1 (2005).

[34] J. Chabe et al., arXiv:0709.4320

[35] J. F. Kanem et al., Phys. Rev. Lett. 98, 083004 (2007).

[36] D. Poletti, G.C. Carlo, and B. Li, Phys. Rev. E 75, 011102 (2007).

[37] A. Kenfack, J.B. Gong, A.K. Pattanayak, in preparation.

[38] The transition to chaos: Quantum manifestations, L.E. Reichl, Springer-Verlag, NY, 2004.

[39] M. Grifoni et al., Phys. Rev. Lett. 89, 146801 (2002); J. B. Majer et al., Phys. Rev. Lett. 90, 056802 (2003). 